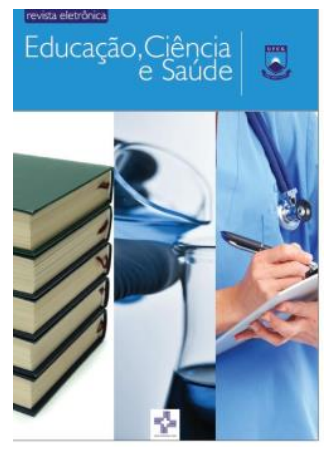

EDUCAÇÃO CIÊNCIA E SAÚDE

http://dx.doi.org/10.20438/ecs.v5i2.149

\title{
O POTENCIAL HEPATOPROTETOR DO GENGIBRE (Zingiber officinale): UMA REVISÃO SOBRE SEUS PRINCIPAIS MECANISMOS DE HEPATOPROTEÇÃO
}

\author{
Paulo Rogério Moreira da Silva ${ }^{1}$, Milena de Cassia Alves Monteiro da Silva ${ }^{1}$, Camila \\ Macena de Oliveira Monteiro ${ }^{1}$, Ennaly Monteiro de Farias ${ }^{1}$, Maria Emília da Silva \\ Menezes $^{2}$ \\ ${ }^{1}$ Curso de Bacharelado em Farmácia, Unidade Acadêmica de Saúde, Universidade Federal de Campina \\ Grande, Cuité-PB, Brasil. \\ ${ }^{2}$ Nutricionista. Doutora em Ciências pela UFAL. Prof ${ }^{\underline{a}}$ Unidade Acadêmica de Saúde, Universidade Federal \\ de Campina Grande, Cuité, PB, Brasil. \\ Email para correspondência: paulormdasilva2012@gmail.com
}

\begin{abstract}
Resumo
O fígado é um órgão vital ao organismo por regular vários processos fisiológicos. Há uma longa tradição em tratar problemas hepáticos com ervas devido a suas propriedades antioxidantes. $O$ Zingiber officinale, conhecido como gengibre, é uma dessas plantas. Visando compreender seus mecanismos hepatoprotetores, foi feita a presente revisão literária. Realizou-se a busca nas bases de dados SciELO, MEDLINE/Pubmed e LILACS, com os termos hepatoprotective e liver combinadas com ginger e Zingiber officinale, analisando-se 16 artigos dos anos 20122018. Os resultados mostram que grande parte dos mecanismos de hepatoproteção associamse à atividade antioxidante por regularem positivamente o fator de transcrição Nfr2 e aumentarem as glutationas hepática, S-transferases (GST), peroxidase (GPx) e catalase (CAT). Há prevenção de cânceres através da diminuição dos genes Caspase3, MK167 e C-fos, e de sua metástase pela inibição do TGF- $\beta 1$. Há redução da expressão de PCNA, colágeno, colesterol e triglicerídeos (por inibição da superexpressão de ChREBP) e alívio da inflamação (por inibição de prostaglandinas, leucotrienos, TNF- $\alpha$, IL-1 $\beta$ e COX-2, e supressão do NF-KB p65, do receptor TLR4 e da MAPK). Tais propriedades relacionam-se com os compostos gingeróis, shagaoles e gingeronas, fazendo do gengibre um produto promissor para a manutenção da integridade do fígado.
\end{abstract}

Palavras-chave: gengibre, estresse oxidativo, fígado, expressão genética.

\begin{abstract}
Liver is a vital organ to the body by regulating various physiological processes. There is a long tradition of treating liver problems with herbs because of their antioxidant properties. Zingiber officinale, known as ginger, is one suchlike plant. In order to understand its hepatoprotective mechanisms, this literary revision was made. We searched the SciELO, MEDLINE/Pubmed and LILACS databases, with the terms hepatoprotective and liver combined with ginger and Zingiber officinale, analyzing 16 articles from years 2012-2018. The results show that most of the hepatoprotection mechanisms are associated with antioxidant activity by positively regulating
\end{abstract}




\begin{abstract}
the transcription factor Nfr2 and increasing hepatic glutathione, S-transferases (GST), peroxidase (GPx) and catalase (CAT). There is cancer prevention through the decrease of Caspase3, MK167 and C-fos genes, and its metastasis by inhibition of TGF- $\beta 1$. There is a reduction in expression of PCNA, collagen, cholesterol and triglycerides (due to inhibition of ChREBP overexpression) and inflammation alleviation (due to inhibition of prostaglandins, leukotrienes, TNF- $\alpha, \mathrm{IL}-1 \beta$ and COX-2, and suppression of NF-KB p65, TLR4 receptor and MAPK). These properties are related to compounds gingerols, shagaoles and zingerones, turning ginger into a promising product for the maintenance of liver integrity.
\end{abstract}

Keywords: ginger, oxidative stress, liver, gene expression.

\title{
1 Introdução
}

O uso de plantas medicinais não é apenas um costume do passado distante. Desse modo, o uso de remédios produzidos a partir de ervas é mais prevalente em pacientes com doenças crônicas. Especiarias e ervas são reconhecidas como fontes de antioxidantes naturais que podem proteger os órgãos do estresse oxidativo, tendo assim, um importante papel na prevenção de doenças cuja etiologia e fisiopatologia estão associadas às espécies reativas do oxigênio - EROs (MOHAMED et al., 2015). O equilíbrio entre EROs e antioxidantes é crucial e pode ser um mecanismo relevante ao prevenir os danos causados pelo estresse oxidativo (JOSHI et al., 2017).

As espécies reativas do oxigênio são comumente relacionadas ao estresse oxidativo, o qual implica em doenças perigosas, dentre elas, doenças cardíacas, diabetes, câncer e, especialmente, lesões hepáticas. O fígado é um órgão vital para o organismo por causa da sua tarefa fundamental em regular vários processos fisiológicos como metabolismo, secreção e armazenamento, e ter uma grande capacidade em desintoxicar substâncias tóxicas e sintetizar substâncias úteis. Por isso, doenças que causam inflamação hepática estão associadas a uma significante taxa de mortalidade e morbidade (ABDEL-AZEEM et al., 2013; CHEONG et al., 2015).

O Zingiber officinale da família Zingiberaceae, conhecido popularmente como gengibre, é uma planta originada do sudeste asiático, mas muito cultivada mundialmente e com vários registros de benefícios à saúde humana (KIM et al., 2017). Seu rizoma em pó trata-se de uma mercadoria importante no mundo inteiro devido ao seu uso como especiaria, influenciando o sabor e aroma de preparações culinárias.

Além disso, o gengibre tem sido utilizado na medicina tradicional no tratamento de problemas gastrintestinais, tais como dores estomacais, espasmos abdominais, náuseas e vômitos. Em relação a seus compostos bioativos, há relatos onde se destaca suas 
propriedades anti-inflamatórias, anticancerígenas, antioxidantes, antihipercolesterolêmicas, anti-hiperlipidêmicas, e antitóxicas (GHOLAMPUR et al., 2017; SALIHU et al., 2015). Dessa forma, o gengibre apresenta propriedades benéficas ao fígado, tanto na prevenção de doenças, quanto no seu tratamento e alívio de sintomas.

Neste trabalho, teve-se em mente que o fígado é um órgão essencial ao organismo, mas bastante suscetível a agressões causadas por vários agentes, tais como metais, toxinas, triglicerídeos e, principalmente, pelo uso de certas drogas. Esclarecer como o gengibre e seus compostos atuam nos hepatócitos, tanto na produção de enzimas antioxidantes, quanto na indução de genes hepatoprotetores, é importante para a criação ou melhoramento de estratégias eficazes que visem preservar a integridade desse órgão vital.

Dessa forma, visando compreender com mais detalhes os mecanismos hepatoprotetores proporcionados pelo gengibre, foi feita a presente revisão literária, na qual se buscou artigos mais recentes a respeito do tema.

\section{Metodologia}

Realizou-se uma revisão literária em busca de artigos, por meio da plataforma Capes, nas bases de dados SciELO, MEDLINE/PubMed e LILACS. Os idiomas priorizados foram inglês e espanhol e utilizou-se os termos hepatoprotective; liver; combinadas com Zingiber officinale ou ginger. Obteve-se 243 resultados na pesquisa. Assim, a fim de diminuir o número de artigos a serem analisados, a sua data de publicação foi delimitada em seis anos, ou seja, na faixa de 2012-2018.

A partir dos títulos e resumos encontrados, excluíram-se aqueles que não comprovavam ou não sugeriam mecanismos de hepatoproteção. Dessa maneira, foram escolhidos 16 artigos para serem analisados na íntegra. Todos esses apresentaram comprovações do potencial hepatoprotetor do gengibre (Zingiber officinale), a maior parte abordando a sua atividade anti-inflamatória e antioxidante perante injúrias acometidas ao fígado. Por essa razão, foram então selecionados para a composição do presente artigo.

\section{Resultados e discussão}

O fígado é um órgão vital para o organismo, por isso doenças e substâncias que causam inflamação hepática estão associadas a uma significante morbidade e mortalidade. Há 
uma longa tradição em tratar problemas hepáticos com drogas botânicas, atuando como agentes terapêuticos, e algumas ervas têm mostrado efeito promissores, tanto em culturas celulares e estudo em animais, quanto em avaliações clínicas (CHEONG et al., 2015).

Plantas medicinais com potencial antioxidante possuem eficácia contra várias doenças humanas. O Zingiber officinale, comumente conhecido como gengibre, pertence à família Zingiberaceae, tendo propriedades anti-inflamatórias, anti-hipercolesterolêmicas, anti-hiperlipidêmicas, antieméticas e antitóxicas, podendo assim atuar como protetor do fígado diante de várias ameaças à sua função e integridade (GHOLAMPUR et al., 2017).

Uma das ameaças que prejudicam o fígado por aumentarem o estresse oxidativo é o acúmulo ou exposição a certos metais, tais como o chumbo, um dos metais pesados poluidores mais abundante no ambiente. A exposição crônica ao elemento resulta, principalmente no fígado, em várias mudanças metabólicas e histológicas, danos na membrana, expressão de genes alterados e apoptose. O chumbo induz a geração de espécies reativas do oxigênio (EROs), aumentando os níveis de peroxidação lipídica e de substâncias reativas ao ácido barbitúrico (TBARs) e inibindo a atividade de biomoléculas antioxidantes (MOHAMED et al., 2015).

Em relação ao chumbo, o uso de extrato de gengibre parece proteger o fígado ao aumentar os níveis de glutationa hepática, glutationa S-transferases (GST), glutationa peroxidase (GPx) e de catalase (CAT), gerando ainda up-regulation na expressão de mRNA de genes antioxidantes, embora não reduza as concentrações do metal (MOHAMED et al., 2015).

Consequências advindas do acúmulo de ferro, cádmio e mercúrio também foram aliviadas com o uso do Z. officinale. Em relação ao ferro (Fe), Gholampur et al. (2017) verificaram que o extrato de gengibre tem seu efeito hepatoprotetor não só devido à propriedade de limpeza de radicais livres, mas também por apresentar um efeito quelato com o elemento, eliminando o seu excesso.

Já Baiomy e Mansour (2015), buscando respostas histopatológicas e genéticas para a toxicidade causada pelo cádmio (Cd), perceberam que, ao administrar gengibre nas refeições de coelhos, havia diminuição na expressão de mRNA de genes apoptóticos (Caspase3), de proliferação (MK167), de proto-oncogenes (C-fos) e antioxidantes (GST), contrabalanceando os efeitos tóxicos do metal. Além disso, o uso do extrato de gengibre previne a degradação oxidativa da membrana proveniente do ataque de radicais livres 
mediados pelo mercúrio $(\mathrm{Hg})$, atuando contra as suas consequentes disfunções hepáticas e renais (JOSHI et al., 2017).

Bardi et al. (2013) relatam a proteção dos extratos contra cirrose e lesões hepáticas. A cirrose hepática trata-se de um processo irreversível caracterizado pela deposição de excesso de matriz extracelular (ECM) acompanhada da formação de cicatrizes e destruição da arquitetura do fígado, podendo assim bloquear o fluxo sanguíneo.

Em laboratório, pode-se usar o produto químico tioacetanamida (TAA) na indução de necrose hepática, pois a substância gera estresse oxidativo durante seu metabolismo pelo microssomo CYP2E1. Principalmente por prevenir a formação de radicais livres, 0 extrato de gengibre diminui a fibrose e reduz a expressão de PCNA - uma proteína nuclear sintetizada na fase $\mathrm{G}_{1} / \mathrm{S}$ do ciclo celular relacionada com a proliferação de células na cirrose (BARDI et al., 2013).

Estudos, como o de Poorrostami, Farokhi e Heidari (2014), comprovam também a capacidade do gengibre em proteger o fígado contra a toxicidade proveniente do uso de drogas. Nessa referida pesquisa, utilizando ratas Wistar tratadas com lamotrigina, os autores sugeriram melhora nas funções hepáticas dos animais ao ingerirem extrato hidroalcoólico de gengibre.

A lamotrigina é uma droga muito utilizada no tratamento de epilepsia e de desordens bipolares, fazendo parte da classe das feniltriazinas. Seu mecanismo de ação consiste no bloqueio de canais de sódio voltagem-dependentes, o qual estabiliza a membrana pré-sináptica e inibe a liberação do neurotransmissor excitatório. Pacientes que recebem tratamento crônico com tal medicamento estão em alto risco de desenvolverem sintomas relacionados à hepatotoxicidade (POORROSTAMI; FAROKHI; HEIDARI, 2014).

Certos fármacos antiepiléticos usados por um grande período de tempo aumentam o estresse oxidativo ao ocasionarem um desequilíbrio na geração de radicais livres e moléculas antioxidantes. Sua consequência mais importante é a peroxidação lipídica, gerando malondialdeído e causando rompimento da membrana celular. $O$ extrato hidroalcoólico de Zingiber officinale atua inibindo as prostaglandinas e a síntese de leucotrienos, protegendo o tecido contra peroxidação lipídica e diminuindo os níveis de colesterol e triglicerídeos. Além disso, assim como a silimarina, diminui a concentração sérica das enzimas transaminases ALT, AST e ALP, e de malondialdeído, indicando a 
estabilização da membrana (ABDEL-AZZEM et al., 2013; GOMÉZ-RODRÍGUEZ; SUÁREZ; IZQUIERDO-SÁNCHEZ, 2013; POORROSTAMI; FAROKHI; HEIDARI, 2014).

Outro fármaco bastante popular e de fácil acesso é o acetaminofeno (paracetamol), cuja superdosagem produz dano hepático. Ele faz parte do grupo dos anti-inflamatórios não-esteroidais (AINES), amplamente utilizado como antipirético e no combate à dor, inibindo as enzimas ciclo-oxigenase-1 (COX-1) e ciclo-oxigenase-2 (COX-2) e, consequentemente, a síntese de prostaglandinas. O potencial antioxidante do extrato hidroalcoólico limita o dano hepático ocasionado pelo acetaminofeno e diminui os níveis enzimáticos de ALT e AST, sugerindo proteção contra a hepatotoxicidade (GOMÉZRODRÍGUEZ; SUÁREZ; IZQUIERDO-SÁNCHEZ, 2013).

Em estudo também com o acetaminofeno, Abdel-Azeem et al. (2013) comparou o mecanismo do $Z$. officinale responsável pelo alívio do estresse oxidativo com o mecanismo antioxidante da vitamina $\mathrm{E}$, no qual há prevenção da produção, neutralização e limpeza de radicais livres, supressão do acúmulo de espécies reativas de oxigênio e nitrogênio, melhora da atividade de superóxido dismutase, glutationa peroxidase e aumento no nível de glutationa reduzida.

Assim como protege o fígado diante de metais e medicamentos hepatotóxicos, o Zingiber officinale exibe essa mesma proteção sobre o acúmulo de gordura hepática, uma doença crônica que deixa o órgão vulnerável a inflamações, podendo resultar em hepatite, fibrose e cirrose hepática, câncer de fígado e consequências ao sistema cardiovascular. Esse acúmulo de triglicerídeos nos hepatócitos pode ser ocasionado pelo consumo excessivo de álcool ou de alimentos ricos em frutose (GAO et al., 2012; LIU et al., 2013; NWOZO; OSUNMADEWA; OYINLOYE, 2014).

No caso da doença hepática gordurosa alcoólica, metabólitos como os Dglucurono-6,3-lactona, glicerol-3-fosfato, ácidos pirúvico, litocólico e 2-pirocatecúcico, e a prostaglandina E1 se elevam, mas seus níveis são restaurados com a administração de óleo essencial de gengibre, diminuindo o processo inflamatório. Além disso, o óleo reduz a atividade de triglicerídeos, do colesterol total, e do malondialdeído hepático, possuindo efeitos protetores melhores do que os do óleo de cúrcuma - Curcuma longa (LIU et al., 2013; NWOZO; OSUNMADEWA; OYINLOYE, 2014).

O excesso de frutose na alimentação desencadeia o acúmulo de gordura no fígado ao ativar as proteínas de ligação do elemento de resposta sensível a carboidratos (ChREBP), o qual age em sinergismo com a proteína de ligação ao elemento de resposta 
a esteróis (SREBP1c) para aumentar a expressão de genes lipogênicos. Embora não altere a expressão da SREBP1c, o gengibre anula a superexpressão da ChREBP, impedindo desse modo a hipertrigliceridemia e a deposição de gordura hepática (GAO et al., 2012).

O referido efeito hepatoprotetor do Zingiber officinale decorre da presença de seus princípios ativos, tais como os gingeroles, shogaoles e gingeronas. 0 6-gingerol, por exemplo, restaura o peso normal do fígado e é responsável pela limpeza de radicais livres em ratos expostos ao carbendazim, um fungicida utilizado na veterinária e na agricultura que prejudica as funções renais e hepáticas (SALIHU et al., 2015).

Pesquisas de Salihu et al. (2015) mostram que ratos afetados pelo carbendazim tiveram diminuição das atividades da superóxido dismutase (SOD), catalase (CAT), glutationa peroxidase (GPX), e glutationa reduzida (GSH), no entanto, a coadministração com o 6-gingerol melhorou a atividade dessas enzimas antioxidantes e do nível de GSH, como também dos níveis de $\mathrm{H}_{2} \mathrm{O}_{2}$ elevados pelo fungicida, restaurando-os à sua normalidade (SALIHU et al., 2015). Em relação a esse princípio ativo, Joshi et al. (2017) sugere sua combinação com o mercúrio inorgânico a fim de melhorar a segurança de vacinas pediátricas.

Os ácidos fenólicos, como o 6-gingerol e o 6-shogaol, também demonstram atividade hepatoprotetora contra o estresse oxidativo induzido pela aflatoxina $\mathrm{B}_{1}$, uma micotoxina contaminante de alimentos que, junto com o vírus da hepatite $B$, é uma das causas da formação de carcinoma hepatocelular na população. Além de inibirem os danos ao DNA causados pelas (EROs), os compostos fenólicos regulam positivamente o fator de transcrição Nfr2, o qual controla a expressão de enzimas antioxidantes importantes para a prevenção da toxicidade gerada pela micotoxina (VIPIN et al., 2017).

Já as gingeronas, existentes em quantidade considerável no gengibre $(9.25 \%)$, exibem efeito antioxidante, anti-inflamatório e hepatoprotetor nas lesões causadas pelo tetracloreto de carbono e pela dimetilnitrosamina. Tais propriedades se devem ao enfraquecimento de mediadores inflamatórios (fator de necrose tumoral $\alpha$, interleucina-1 $\beta$, ciclooxigenase-2) e à supressão da ativação do fator nuclear k-B p65, além da diminuição do acúmulo de colágeno que estão elevados durante as lesões. É importante ressaltar que a concentração de gingeronas é maior quando o gengibre está seco ou torrado (CHEONG et al., 2015). 
Ainda sobre as gingeronas, Kim et. al. (2017) relata seu potencial em suprimir a metástase do carcinoma hepatocelular e a falha hepática induzida por polissacarídeos. $\mathrm{O}$ carcinoma hepatocelular é uma das causas mais comuns de morbidade e mortalidade na Ásia e na África, sendo associada a infecções virais, consumo excessivo de álcool, oncogenes transgênicos e outros indutores de cirrose hepática.

A metástase desse carcinoma pode ser ocasionada devido à reativação da transição epitelial-mesenquimal, um processo no qual as células epiteliais perdem seus fenótipos epiteliais e adquirem características mesenquimais e que pode ser induzido pelo fator de crescimento- $\beta 1$ (TGF- $\beta 1$ ). O uso sinérgico da gingerona com uma molécula derivada da mesma, inibiu essa transição ao suprimir a expressão de E-caderinas e aumentar a de N-caderinas em células de carcinoma hepatocelular ativadas pelo TGF- $\beta 1$, impedindo a migração e invasão das mesmas (KIM et al., 2017).

Em relação à falha hepática induzida por polissacarídeos, a gingerona injetada por via intravenosa suprimiu o aumento dos níveis séricos de ALT e AST e normalizou a arquitetura celular e lobular de tecidos hepáticos, sugerindo proteção contra lesões letais do fígado, bem como inibiu o aumento do receptor TLR4 e a sinalização da proteína quinase ativada por mitógeno (MAPK), relacionados à indução de respostas inflamatórias. Dessa maneira, a gingerona representa uma potencial intervenção farmacológica, sendo necessário 50,4 mg do princípio ativo em um adulto de $70 \mathrm{~kg}$ para que o composto possa exibir efeitos hepatoprotetores quando administrado intravenosamente (LEE et al., 2018).

\section{Conclusão}

A partir dos resultados encontrados na presente revisão literária, os mecanismos de ação expressos pelo gengibre - Zingiber officinale - em relação à sua atividade hepatoprotetora foram esclarecidos. Tal propriedade se deve principalmente à limpeza de radicais livres e melhora na atividade de enzimas antioxidantes.

Além disso, o gengibre participa da regulação da expressão de genes e de proteínas cancerígenas, tendo também papel importante no controle da inflamação e no acúmulo de substâncias tóxicas ao fígado, como drogas, metais e excesso de gordura.

Tudo isso se deve à presença de princípios ativos em sua composição, como os gingeróis, shagaoles e gingeronas. Dessa maneira, o gengibre se revela como um produto em potencial, tanto no tratamento e prevenção de doenças que acometem o 
fígado, quanto para a síntese de fármacos ou isolamento de compostos de atividade biológica.

\section{$5 \quad$ Referências}

ABDEL-AZEEM, Amal S et al. Hepatoprotective, antioxidant, and ameliorative effects of ginger (Zingiber officinale Roscoe) and vitamin E in acetaminophen treated rats. Journal Of Dietary Supplements, Cairo, v. 10, n. 3, p.195-209, ago. 2013. Informa UK Limited. Disponível em: $<$ http://citeseerx.ist.psu.edu/viewdoc/download?doi=10.1.1.870.876\&rep=rep1\&type=pdf>. Acesso em: 09 fev. 2018.

BAIOMY, Ahmed A.; MANSOUR, Ahmed A. Genetic and Histopathological Responses to Cadmium Toxicity in Rabbit's Kidney and Liver: Protection by Ginger (Zingiber officinale). Biological Trace Element Research, [s.I.], v. 170, n. 2, p.320-329, 3 set. 2015. Springer Nature. Disponível em: <https://link.springer.com/article/10.1007/s12011-015-0491-4>. Acesso em: 26 dez. 2017.

BARDI, Daleya Abdulaziz et al. In Vivo Evaluation of Ethanolic Extract of Zingiber officinale Rhizomes for Its Protective Effect against Liver Cirrhosis. Biomed Research International, Kuala Lumpur, v. 2013, p.1-10, 2013. Hindawi Limited. Disponível em: <https://www.hindawi.com/journals/bmri/2013/918460/abs/>. Acesso em: 26 dez. 2017.

CHEONG, Kyoung Ook et al. Hepatoprotective effects of zingerone on carbon tetrachloride- and dimethylnitrosamine-induced liver injuries in rats. Archives Of Pharmacal Research, [s.I.], v. 39, n. 2, p.279-291, 14 dez. 2015. Springer Nature. Disponível em: <https://link.springer.com/article/10.1007/s12272-015-0696-2>. Acesso em: 09 fev. 2018.

GAO, Huanqing et al. Treatment with Ginger Ameliorates Fructose-Induced Fatty Liver and Hypertriglyceridemia in Rats: Modulation of the Hepatic Carbohydrate Response Element-Binding Protein-Mediated Pathway. Evidence-based Complementary And Alternative Medicine, [s.I.], v. 2012, p.1-12, 2012. Hindawi Limited. Disponível em: <https://www.hindawi.com/journals/ecam/2012/570948/>. Acesso em: 04 jan. 2018.

GHOLAMPOUR, Firouzeh et al. The protective effect of hydroalcoholic extract of Ginger (Zingiber officinale Rosc.) against iron-induced functional and histological damages in rat liver and kidney. Avicenna Journal of Phytomedicine, Shiraz, v. 7, n. 6, p. 542-553, nov./dez. 2017. Disponível 
em: <http://ajp.mums.ac.ir/article_9113_aa5b6d082c2ea61eda154515969892d7.pdf>. Acesso em: 26 nov. 2017.

GÓMEZ-RODRÍGUEZ, Banely Trinidad; CORTÉS SUÁREZ, Saúl; IZQUIERDO-SÁNCHEZ, Teresa. Efecto del extracto hidroalcohólico de Zingiber officinale Roscoe (jengibre) en modelo de hepatotoxicidad en ratas. Revista Cubana de Plantas Medicinales, v. 18, n. 3, p.431-444, 2013. Disponível em: <http://scielo.sld.cu/pdf/pla/v18n3/pla10313.pdf> Acesso em: 27 dez. 2017.

JOSHI, Deepmala et al. Zingiber officinale and 6-gingerol alleviate liver and kidney dysfunctions and oxidative stress induced by mercuric chloride in male rats: A protective approach. Biomedicine \& Pharmacotherapy, [s.I.], v. 91, p.645-655, jul. 2017. Elsevier BV. Disponível em: <https://www.sciencedirect.com/science/article/pii/S0753332217314014>. Acesso em: 02 fev. 2018.

KIM, Young-joo et al. Combined treatment with zingerone and its novel derivative synergistically inhibits TGF- $\beta 1$ induced epithelial-mesenchymal transition, migration and invasion of human hepatocellular carcinoma cells. Bioorganic \& Medicinal Chemistry Letters, [s.l.], v. 27, n. 4, p.1081-1088, fev. 2017. Elsevier BV. Disponível em: <http://www.sciencedirect.com/science/article/pii/S0960894X16313117?via=ihub>. Acesso em: 29 dez. 2017.

LEE, Wonhwa et al. Protective effects of zingerone on lipopolysaccharide-induced hepatic failure through the modulation of inflammatory pathways. Chemico-biological Interactions, [s.I.], v. 281, p.106-110, fev. 2018. Elsevier BV. Disponível em: <https://www.sciencedirect.com/science/article/pii/S000927971731147X?via=ihub>. Acesso em: 09 fev. 2018.

LIU, Chun-ting et al. Metabolomics of Ginger Essential Oil against Alcoholic Fatty Liver in Mice. Journal Of Agricultural And Food Chemistry, Taipei, v. 61, n. 46, p.11231-11240, 12 nov. 2013. American Chemical Society (ACS). Disponível em: <http://pubs.acs.org/doi/10.1021/jf403523g>. Acesso em: 05 jan. 2018.

MOHAMED, Omnia Ismail et al. Ginger extract modulates $\mathrm{Pb}$-induced hepatic oxidative stress and expression of antioxidant gene transcripts in rat liver. Pharmaceutical Biology, [s.I.], p.1-9, 16 jun. 2015. Informa LK Limited. Disponível em: <http://www.tandfonline.com/doi/full/10.3109/13880209.2015.1057651>. Acesso em: 27 dez. 2017. 
NWOZO, Sarah Onyenibe; OSUNMADEWA, Damilola Adeola; OYINLOYE, Babatunji Emmanuel. Anti-fatty liver effects of oils from Zingiber officinale and Curcuma longa on ethanol-induced fatty liver in rats. Journal Of Integrative Medicine, [s.I.], v. 12, n. 1, p.59-65, jan. 2014. Elsevier BV. Disponível em: <https://www.sciencedirect.com/science/article/pii/S2095496414600066>. Acesso em: 02 fev. 2018.

POORROSTAMI, Ameneh; FAROKHI, Farah Farokhi; HEIDARI, Reza. Effect of hydroalcoholic extract of ginger on the liver of epileptic female rats treated with lamotrigine. Avicenna Journal Of Phytomedicine, Urmia, v. 4, n. 4, p.276-286, jul. 2014. Mashhad University of Medical Sciences. Disponível em: <https://www.ncbi.nlm.nih.gov/pmc/articles/PMC4110781/>. Acesso em: 05 jan. 2018.

SALIHU, Mariama et al. 6-gingerol-rich fraction from Zingiber officinale prevents hematotoxicity and oxidative damage in kidney and liver of rats exposed to carbendazim. Journal Of Dietary Supplements, Ibadan, v. 13, n. 4, p.433-448, 16 dez. 2015. Informa UK Limited. Disponível em: <http://www.tandfonline.com/doi/full/10.3109/19390211.2015.1107802?scroll=top\&needAccess=tru e>. Acesso em: 29 dez. 2017.

VIPIN, A. V. et al. Protective effects of phenolics rich extract of ginger against Aflatoxin B 1 induced oxidative stress and hepatotoxicity. Biomedicine \& Pharmacotherapy, Karnataka, v. 91, p.415-424, jul. 2017. Elsevier BV. Disponível em: <http://www.sciencedirect.com/science/article/pii/S0753332217307941 ?via=ihub>. Acesso em: 27 dez. 2017. 